\title{
Diagnostic Accuracy of CT-Guided Core Biopsy of Ground-Glass Opacity Pulmonary Lesions
}

\begin{abstract}
Tae Jung Kim ${ }^{1}$
Jae-Ho Lee ${ }^{2}$

Choon-Taek Lee ${ }^{2}$

Sang Hoon Jheon ${ }^{3}$

Sook Whan Sung ${ }^{3}$

Jin-Haeng Chung ${ }^{4}$

Kyung Won Lee ${ }^{1}$
\end{abstract}

Keywords: core biopsy, CT, diagnostic accuracy, ground-glass opacity, lung

DOI:10.2214/AJR.07.2441

Received April 18, 2007; accepted after revision July 23, 2007.

'Department of Radiology, Seoul National University Bundang Hospital; Seoul National University College of Medicine; and Institute of Radiation Medicine, Seoul National University Medical Research Center; 300 Gumi-dong, Bundang-gu, Seongnam-si, Gyeonggi-do 463-707, Korea. Address correspondence to K. W. Lee (lkwrad@radiol.snu.ac.kr).

${ }^{2}$ Department of Medicine, Seoul National University Bundang Hospital and Seoul National University College of Medicine, Gyeonggi-do, Korea.

${ }^{3}$ Department of Thoracic Surgery, Seoul National University Bundang Hospital and Seoul National University College of Medicine, Gyeonggi-do, Korea.

${ }^{4}$ Department of Pathology, Seoul National University Bundang Hospital and Seoul National University College of Medicine, Gyeonggi-do, Korea.

AJR2008; 190:234-239

$0361-803 X / 08 / 1901-234$

(C) American Roentgen Ray Society

OBJECTIVE. The purpose of our study was to evaluate the accuracy of CT-guided percutaneous core biopsy of ground-glass opacity (GGO) pulmonary lesions.

MATERIALS AND METHODS. The study included 50 patients (24 men, 26 women; age range, 43-78 years) who had a GGO pulmonary lesion and underwent CT-guided core biopsy. Diagnostic accuracy was compared between two groups according to lesion size $(<2$ $\mathrm{cm}$ vs $\geq 2 \mathrm{~cm}$ ) and GGO component (>90\% vs 50-90\%). Each case was reviewed for complications, including pneumothorax, thoracostomy tube insertion, and hemoptysis.

RESULTS. Malignancy was finally diagnosed in 33 patients, including three who underwent repeated biopsies, with 33 true-positive and three false-negative findings for an overall sensitivity of $92 \%$ (33/36). A benign lesion was finally diagnosed in 10 patients with one false-positive result, for a specificity of $90 \%$. Two benign lesions without confirmative diagnosis because of loss of follow-up and five nondiagnostic samples were excluded from the calculations of sensitivity, specificity, and diagnostic accuracy. The overall diagnostic accuracy was $91 \%$, with a positive predictive value of $97 \%$ and a negative predictive value of $75 \%$. Sensitivity and accuracy were not significantly different between the two groups of lesion size and GGO components $(p=0.0491)$. Ten $(18 \%)$ patients had pneumothorax, with one $(2 \%)$ requiring placement of a thoracostomy tube. Mild hemoptysis occurred in seven $(13 \%)$ patients.

CONCLUSION. CT-guided core biopsy of GGO lesions can yield high diagnostic accuracy and acceptable complication rates approaching those of solid lesions.

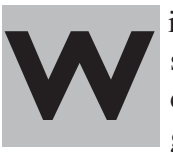
ith recent advances in $\mathrm{CT}$ screening for lung cancer, an increase in the detection of ground-glass opacity (GGO) lesions has been noted [1-3]. GGO is a nonspecific finding that may be caused by various disorders, including inflammatory diseases, focal fibrosis, atypical adenomatous hyperplasia, bronchoalveolar carcinoma (BAC), and adenocarcinoma [4-6]. The clinical significance of localized GGO is its high incidence of malignancy compared with solid nodules $[7,8]$. The approach to the diagnosis of localized GGO is often difficult. Currently, most cases of localized GGO detected at initial CT are reevaluated with follow-up CT after a 2or 3-month interval. Whether lung biopsy should be considered for persistent GGO remains controversial [9].

Percutaneous transthoracic needle biopsy is a well-established method in the histologic diagnosis of pulmonary nodules, and the accuracy of diagnosis of benign and malignant disease is greater than $80 \%$ and $90 \%$, respectively [10-16]. However, to our knowledge, the accuracy of CT-guided core biopsy of GGO lesions has not been reported in detail. Thus, the purpose of this study was to evaluate the diagnostic accuracy of CTguided core biopsy of GGO lesions.

\section{Materials and Methods}

Our institutional review board approved this study and waived informed patient consent.

\section{Study Population and Biopsy Indication}

We retrospectively reviewed the medical and imaging records of all patients who underwent CT-guided needle biopsy of pulmonary nodules at our institution between June 1, 2003, and April 30, 2006. Two chest radiologists who had 8 and 12 years of experience in chest $\mathrm{CT}$ interpretation identified GGO lesions on CT by consensus. Images were displayed with a lung window setting (center, $-600 \mathrm{H}$; width, 1,500 H). The GGO components were semiquantitatively classified 
according to the area they occupied in the whole lesion. GGO was defined as an area of increased attenuation without obscuration of the underlying vessels and bronchi [17]. The percentage of $D_{G G O}$ was calculated as follows: $\left(\left[D_{G G O}-D\right] / D_{G G O}\right.$ ) $\times 100$, where $D_{G G O}$ is the greatest diameter of the lesion, including the GGO area, and $D$ is the greatest diameter of the lesion without GGO. GGO lesions were classified into two groups according to the lesion diameter $(<2 \mathrm{~cm}$ vs $\geq 2 \mathrm{~cm})$ and the percentage of the GGO component (>90\% vs 50 $90 \%$ ). Lesions with a GGO component less than $50 \%$ were excluded from this study because these lesions are mainly solid in nature.

The study included 50 patients ( 24 men, 26 women; age range, 43-78 years; mean age, 61 years); 29 patients found during lung cancer screening studies and 21 patients found during studies for clinical reasons. The mean lesion diameter was $1.9 \pm 0.9(\mathrm{SD}) \mathrm{cm}$ (range, 0.7-4.5 $\mathrm{cm})$. Three lesions were rebiopsied because of a radiologic suspicion of malignancy. A repeat biopsy was considered a new procedure for purposes of calculating diagnostic accuracy.

In our institution, we perform follow-up CT for initially detected GGO lesions after a 2- or 3-month interval. The biopsy method for the persistent GGO lesion (i.e., CT-guided vs surgical biopsy) is determined on an individual basis considering radiologic and clinical features and the views of the patient. All biopsies were performed at the request of pulmonary physicians or surgeons for persistent and localized GGO lesions after an observation period of several months (mean, 2.4 months).

\section{Biopsy Procedures and Histologic Diagnosis}

The biopsies were performed by experienced chest radiologists who had 5 and 9 years of experience performing thoracic biopsy. Written informed consent was obtained from each patient before each biopsy. All biopsies were performed under CT guidance using a 16-MDCT scanner (MX 8000 IDT, Philips Medical Systems). At the time of biopsy, selected images were obtained in the area of interest with 3-mm-thick contiguous transverse CT sections. Biopsies were planned to avoid visible bullae and to cross the fewest pleural surfaces. The procedure was performed with the patient in a prone, supine, or lateral decubitus position, depending on the location of the lesion. Local anesthesia was administered by means of a subcutaneous injection of $1 \%$ lidocaine (Xylocaine, AstraZeneca). A core biopsy specimen was obtained with a detachable 18- or 20-gauge automated cutting needle (Acecut, TSK Laboratory). Using intermittent CT scans to evaluate the needle trajectory, the needle was inserted through the pleura and advanced to a position close to a target lesion. After the position of the needle tip position was confirmed on CT scans, the automated needle biopsy system was fired. Decisions to perform additional needle passes were based on whether, on visual inspection, the specimen was deemed safe and feasible by the radiologists. Core specimens were immersed in $10 \%$ formalin for pathologic examination and diagnosis by several pathologists. The histologic categories used for diagnosis of adenocarcinoma included BAC, adenocarcinoma with BAC features, and adenocarcinoma that was not bronchoalveolar. Cases classified as BAC fit the 2004 World Health Organization description of BAC; namely, an adenocarcinoma with a pure bronchoalveolar growth pattern and no evidence of stromal, vascular, or pleural invasion [18].

\section{Postprocedure Imaging and Care}

After biopsy, CT images were obtained to verify the appearance of the lung and to check for immediate pneumothorax and hemorrhage. The patients were monitored in a ward and positioned with the puncture side down. A posteroanterior expiratory chest radiograph with the patient erect was obtained 3 hours after biopsy. If a small asymptomatic pneumothorax developed, the patient was treated conservatively with monitoring of vital signs, administration of supplemental oxygen, and follow-up chest radiography 1 hour later to evaluate the stability of the pneumothorax. Patients with pneumothorax that was enlarging or accompanied by symptoms of shortness of breath, pain, or low oxygen saturation received chest tubes. Chest tubes were placed by the chest radiologist who performed the biopsy.

\section{Data Collection and Statistical Analysis}

The medical and imaging records of all patients were reviewed by two chest radiologists, and data were collected concerning size and GGO component of the lesions, length of the needle path (i.e., length of the aerated lung traversed by the needle from the surface of the pleura to the proximal margin of the target lesion), number of pleural punctures, results of histopathologic and microbiologic examinations of the biopsy specimen, and complications. Agreement between results of the core needle and surgical biopsies was evaluated.

The sensitivity, specificity, positive and negative predictive values, and diagnostic accuracy were also calculated for patients, with subsequent follow-up data on the basis of clinical and imaging follow-up and review of medical records for at least 12 months after biopsy. The pathologic results of needle biopsy and surgery were reviewed and divided into diagnostic categories that included malignant or premalignant (atypical adenomatous hyperplasia), benign, and nondiagnostic. A designation of a malignant or premalignant lesion was considered a positive result.

A positive biopsy result was considered to be true-positive when there was surgical confirmation or when the results of biopsy of another site revealed malignancy with the same histologic characteristics. A positive biopsy result was considered to be false-positive when there was no evidence of malignancy at surgical resection without preoperative chemotherapy or when there was nodule regression at follow-up CT in the absence of therapy. Designation of a benign lesion was considered a negative result. A negative biopsy result was considered to be true-negative when there was a definitive diagnosis of a benign entity, such as pneumonia or tuberculosis, or when the lesion subsequently disappeared or decreased in size at follow-up CT. A negative result was considered to be false-negative when there was surgical resection with a diagnosis of malignancy.

We compared the results between the two groups according to lesion size $(<2 \mathrm{~cm}$ vs $\geq 2 \mathrm{~cm})$ and GGO component (> 90\% vs 50-90\%): sensitivity, specificity, diagnostic accuracy, incidence of adequate samples for histopathologic analysis, and lesion depth. Diagnostic accuracy was calculated by comparing core needle biopsy results with the final diagnosis based on postsurgical pathologic examinations $(n=34)$ or follow-up imaging $(n=9)$. Procedures in which the tissue specimens were deemed nondiagnostic $(n=5)$ and in which a definite diagnosis of benignancy or malignancy could not be established $(n=2)$ because of loss of follow-up were excluded from the calculation of sensitivity, specificity, and diagnostic accuracy. Fisher's exact test was used to assess the statistical significance of the difference in diagnostic accuracy between the two biopsy groups according to the lesion size and the GGO component, respectively. An unpaired Student's $t$ test was used to assess the statistical significance of the difference between the two groups according to the GGO component. A value for $p$ of less than 0.05 was considered to indicate statistical significance.

\section{Results}

Malignancy or premalignant lesions identified at CT-guided core biopsy underwent surgical resection for histologic confirmation and treatment. Eighteen patients with adenocarcinoma with a BAC feature on core biopsy were confirmed as having the same histology at surgical resection. In 13 patients diagnosed as having BAC at core biopsy, seven patients were confirmed to have adenocarcinoma with a BAC feature, and six patients were confirmed to have BAC after surgical 
Kim et al.

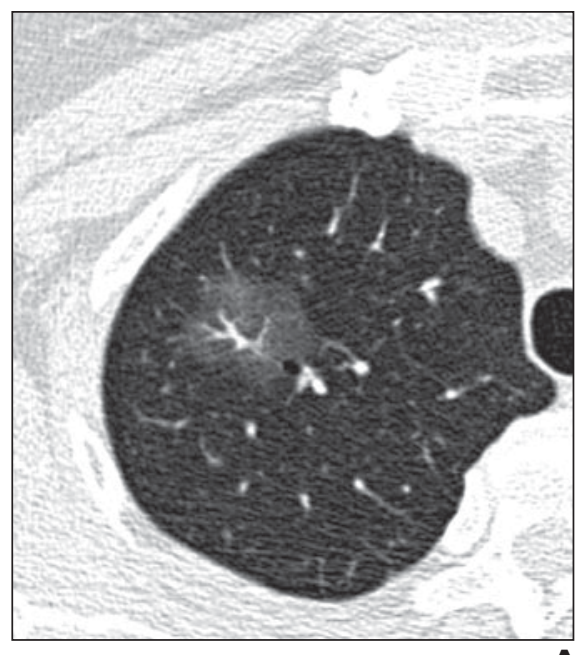

A
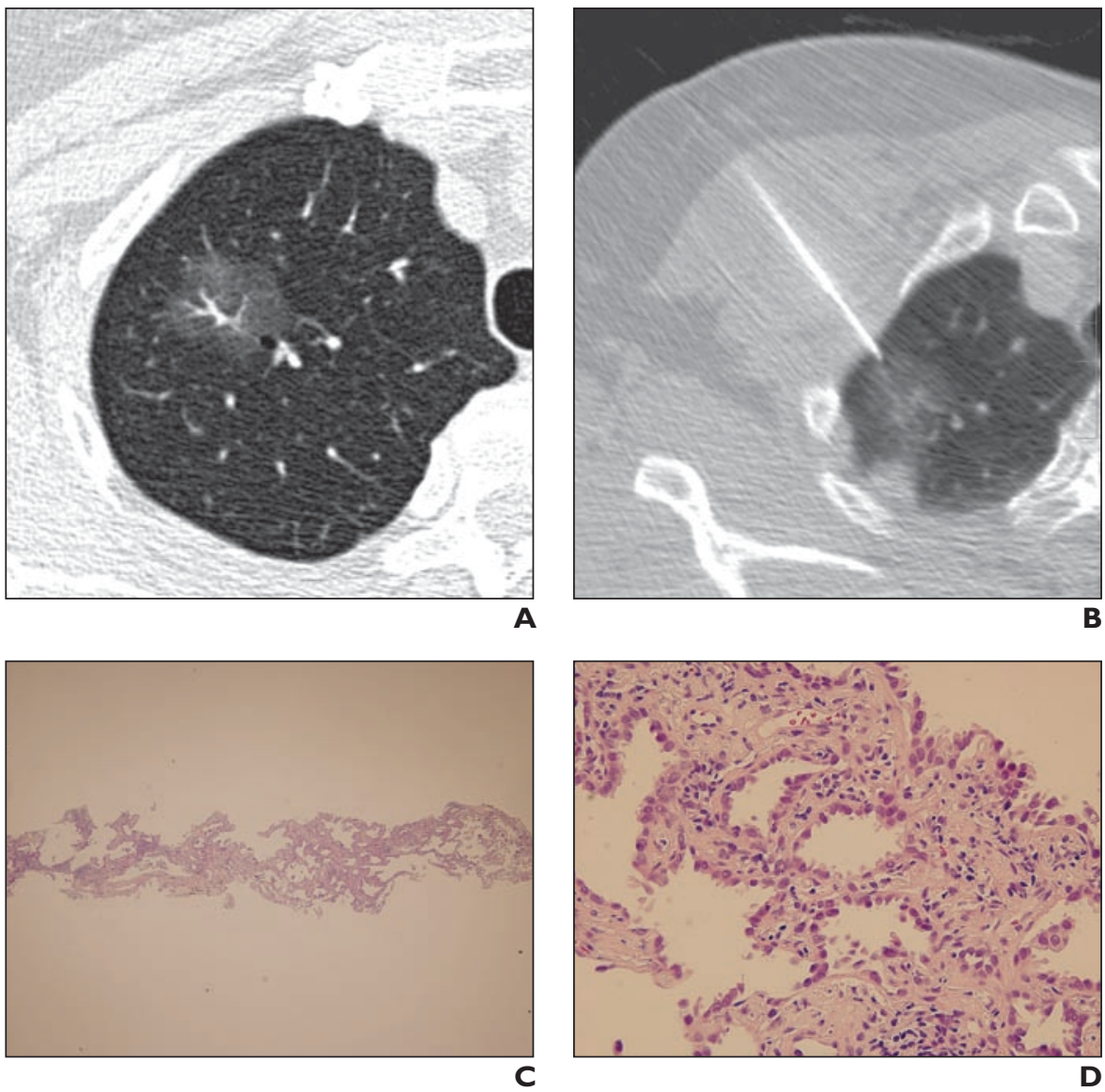

Fig. 1-CT-guided core biopsy in 60-year-old woman with pure ground-glass opacity (GG0) lesion in right upper lobe.

A, High-resolution CT scan shows 3-cm pure GGO lesion in right upper lobe.

B, CT scan obtained during biopsy shows biopsy needle targeting GGO lesion.

C. Photomicrograph shows gross core biopsy specimen. $(\mathrm{H}$ and $\mathrm{E}, \times 10)$

D, Photomicrograph reveals that alveolar walls are lined by cellular proliferation of atypical pneumocytes with hobnail morphology. No evidence of stromal invasion is seen. Histologic diagnosis of core biopsy was bronchoalveolar carcinoma. Final diagnosis after surgical resection was bronchoalveolar carcinoma, Noguchi type A. ( $H$ and $E, \times 200)$

resection (Fig. 1). One patient diagnosed as having atypical adenomatous hyperplasia at core biopsy was confirmed to have BAC after surgical resection. One patient had findings compatible with histologic findings in a preexisting primary malignancy (goblet cell carcinoid tumor of the appendix).

A benign finding was identified in 12 patients; of these, two samples were not confirmed because of loss of findings. Two patients were diagnosed with pneumonia on chest radiography or $\mathrm{CT}$ at 12 - and 18-month follow-up periods, respectively. Two patients were diagnosed with interstitial fibrosis and two with eosinophilic pneumonia. One patient who showed interstitial pneumonia at core biopsy was confirmed to have interstitial pneumonia at surgical biopsy. One patient who was diagnosed with atypical adenoma- tous hyperplasia at core biopsy was found to have chronic interstitial fibrosis at surgical resection. In the remaining two patients, one was confirmed to have tuberculosis and the other was confirmed to have organizing pneumonia at core biopsy. These lesions showed regression at follow-up imaging.

Malignancy or a premalignant lesion was finally diagnosed in 33 patients, including three repeated biopsies, with 33 true-positive and three false-negative findings and an overall sensitivity of $92 \%$ (33/36). A benign lesion was finally diagnosed in 10 patients with one false-positive result (one case of atypical adenomatous hyperplasia on the needle biopsy specimen), generating a specificity of $90 \%$. The overall diagnostic accuracy was $91 \%$, with a positive predictive value of $97 \%$ and a negative predictive value of $75 \%$. Five of
53 biopsy specimens did not allow diagnosis (i.e., nondiagnostic samples); thus, the percentage of diagnostic samples at core biopsy was $91 \%$. Repeated biopsies for the same lung lesion were performed on three patients. Initial biopsy results were suggestive of benign or atypical findings, and second biopsy results were adenocarcinoma $(n=1)$ and $\mathrm{BAC}(n=$ $2)$, respectively. These three patients were confirmed as adenocarcinoma with BAC features $(n=2)$ and $\mathrm{BAC}(n=1)$, respectively, at surgical resection. The final diagnoses of 50 GGO lesions are summarized in Table 1.

Results for lesions $\geq 2 \mathrm{~cm}$ in diameter $(n=$ 23; sensitivity, 94\% [17/18]; accuracy, 96\% [22/23]) were not statistically different from those for lesions $<2 \mathrm{~cm}$ in diameter $(n=$ 23; sensitivity, 94\% [17/18]; accuracy, 91\% [20/22] $)(p=1.0)$. All five nondiagnostic samples were obtained in patients with lesions $<2 \mathrm{~cm}$ in diameter $(p=0.0491)$. The lesion depths were not statistically different between the two groups ( $p=0.4082)$ (Table 2$)$. Results for lesions with GGO $>90 \%(n=21$; sensitivity, 93\% [13/14]; accuracy, 91\% [19/21]) were not statistically different from those for lesions with a GGO of $50-90 \%(n=25$; sensitivity, 91\% [20/22]; accuracy, 92\% [23/25]) $(p=1.0)$. Nondiagnostic samples were obtained in one of 25 lesions with 50-90\% GGO components and four of 21 lesions with $>90 \%$ GGO components $(p=0.1628)$. The mean diameter of the lesions with a $50-90 \%$ GGO area (mean, $2.2 \pm 0.9 \mathrm{~cm}$ ) was larger than that of the lesions with a $>90 \%$ GGO area (mean, $1.6 \pm 0.7 \mathrm{~cm})(p=0.0072)$. Lesion depths were not statistically different between the two groups ( $p=0.3343$ ) (Table 3 ). Most biopsy procedures were completed with one pleural puncture, except for two cases with an additional puncture.

Postbiopsy pneumothorax was seen in 10 (18\%) patients on chest radiography 3 hours after biopsy, and one patient required thoracostomy tube placement. Alveolar hemorrhage along the needle path or around the target lesion developed in 13 patients (23\%) with no major clinical sequelae. Mild hemoptysis occurred in seven patients (13\%). No patient with pulmonary hemorrhage or hemoptysis required treatment.

The overall concordance rate between core and surgical biopsies in malignant and premalignant lesions was $73 \%$ (Table 4). In seven cases the core biopsy specimen did not identify the area of invasion, and the lesions were subsequently diagnosed as adenocarcinoma with BAC features after surgical resec- 
CT-Guided Biopsy of Pulmonary Lesions

TABLE I: Final Diagnoses of 50 Lesions with GroundGlass Opacity

\begin{tabular}{l|c}
\hline Diagnosis & No. of Lesions \\
\hline Malignant lesions & \\
\hline Adenocarcinoma with $_{\text {BAC feature }}^{\text {a }}$ & 25 \\
BAC $^{\text {b }}$ & 7 \\
Metastasis $^{\text {Total }}$ & 1 \\
\hline Benign lesions & 33 \\
\hline Pneumonia & 2 \\
Interstitial fibrosis & 2 \\
Eosinophilic pneumonia & 2 \\
Interstitial pneumonia & 1 \\
Tuberculosis & 1 \\
Organizing pneumonia & 1 \\
Chronic inflammation & 1 \\
Total & 10 \\
\hline Indeterminate lesions & \\
\hline Chronic active inflammation & 1 \\
Type Il pneumocyte & 1 \\
hyperplasia with fibrosis & 2 \\
\hline Total & 5 \\
\hline Nondiagnostic samples & \\
\hline Total & \\
\hline Note BAC & \\
\hline
\end{tabular}

Note-BAC = bronchoalveolar carcinoma

ancludes one repeated biopsy.

${ }^{b}$ Includes two repeated biopsies.

cPatients lost to follow-up.

tion. Two patients with atypical adenomatous hyperplasia at core biopsy were confirmed to have BAC and chronic inflammation, respectively, after surgical resection.

\section{Discussion}

With recent advances in CT screening, the detection of small peripheral lung cancer has been increasing. Most CT-detected lung cancers are adenocarcinomas, which frequently show GGO on CT [1-3]. There has been much interest in the CT features and pathology of GGO, and much has been learned from the correlation of CT images with histology [7, 19-22]. The diagnosis and treatment of GGO have become important issues; however, there is no generally accepted guideline for the management of GGO. Most patients with GGO detected on initial CT underwent follow-up CT after 2- to 3-month intervals [23]. Patients with persistent GGOs usually undergo interventional procedures, such as CT-guided biopsy or video-assisted thoracoscopic surgery

TABLE 2: Diagnostic Yield and Accuracy Based on Lesion Size

\begin{tabular}{l|c|c|c}
\hline \multirow{2}{*}{\multicolumn{1}{c|}{ Variable }} & \multicolumn{2}{|c|}{ Greatest Diameter } & \multirow{2}{*}{} \\
\cline { 2 - 3 } & $\geq 2 \mathrm{~cm}$ & $<2 \mathrm{~cm}$ & 1.0 \\
\hline Sensitivity $(\%)^{\mathrm{a}}$ & $94(17 / 18)$ & $94(17 / 18)$ & 1.0 \\
Accuracy $(\%)^{\mathrm{a}}$ & $96(22 / 23)$ & $91(21 / 23)$ & 0.4082 \\
Lesion depth (mm) & 18 & 21 & 0.0491 \\
No. of nondiagnostic samples & 0 & 5 & \\
\hline
\end{tabular}

${ }^{a}$ Numbers of patients used to calculate percentages are in parentheses. Diagnostic yield and accuracy were calculated on basis of 46 biopsy results, excluding five nondiagnostic samples and two samples without confirmative diagnosis due to loss of follow-up.

(VATS), for the establishment of a histologic diagnosis, which depends on the expertise and equipment of each institute [23-25].

Percutaneous transthoracic needle biopsy with $\mathrm{CT}$ guidance has been shown to be a relatively safe and accurate method for establishing the diagnosis of pulmonary lesions. Its diagnostic accuracy has been reported as greater than $80 \%$ for benign disease and greater than $90 \%$ for malignant disease [10-16]. The overall diagnostic accuracy, sensitivity, and specificity of CT-guided core biopsy in this study are similar to those obtained in previous studies [10-16, 26], although the previous results were obtained from solid nodules. For GGO lesions, to our knowledge, there is only one previous study in which the diagnostic accuracy of CTguided needle aspiration was evaluated [25]. Those authors compared the diagnostic accuracy of CT-guided needle aspiration between the GGO and solid lesions smaller than $2 \mathrm{~cm}$, and suggested that the diagnostic accuracy of CT-guided needle aspiration for GGO lesions was less than that for solid lesions. The lower accuracy was probably due to the low cellularity of GGO lesions, which may frequently result in an inadequate aspiration of air or blood. In addition, the cytologic diagnosis of $\mathrm{BAC}$ is difficult with aspiration biopsy specimens compared with those of a solid tumor. The cytologic features of BAC in aspiration biopsy specimens may merge with those of reactive type 2 pneumocyte hyperplasia and with those of borderline lesions such as sclerosing hemangioma [27, 28].

Previous studies have reported various factors affecting the diagnostic accuracy of CTguided needle aspiration and biopsy of lung nodules [11, 12, 16, 28-34]. Although the size and type of needle used in needle aspiration and biopsy were unlikely to influence the diagnostic sensitivity for lung cancer [29, 35-38], these factors has been reported to affect the diagnostic sensitivity for benign diseases and lymphoma [37, 39]. We performed CT-guided core biopsy using an automated gun that can obtain core specimens to overcome these limitations of the needle aspiration of GGO lesions. The higher accuracy in our study might be due to the use of core biopsy, which can obtain adequate core specimens rather than the scanty cellular aspirates from GGO lesions.

Although our results showed a concordance rate of $73 \%$ in malignant and premalignant lesions, there were several cases of discrepancy between the needle and surgical biopsy results; for seven BACs at core biopsy, the area of invasion was not identified. Given the requirement for BAC to show pure lepidic growth without invasion and the knowledge that most lung adenocarcinomas with a BAC component also have an area of invasion, it is impossible to make an unequivocal diagnosis of BAC in small biopsy specimens [21, 40]. Thus, the correlation with thin-section CT images is warranted when the results of core biopsy suggest $\mathrm{BAC}$, and the entire lesion should be removed to exclude invasion before a final diagnosis of BAC can be made.

When comparing diagnostic accuracy according to lesion size, no statistically significant difference was seen. However, all five nondiagnostic samples were obtained in lesions smaller than $2 \mathrm{~cm}$. This result is comparable to the findings of other reports in the literature [14, 30-33]. In a study of diagnostic accuracy of CT-guided aspiration biopsy classifying lesions into two groups by size, Tsukada et al. [16] reported that the accuracy was $76.4 \%$ for lesions $\leq 2 \mathrm{~cm}$ in diameter and $89.4 \%$ for those $>2 \mathrm{~cm}$ in diameter, which shows the difficulty of obtaining an accurate diagnosis with smaller lesions.

To our knowledge, there has been no report regarding the diagnostic accuracy of core biopsy of GGO lesions according to the GGO components. Contrary to the general expectation that it is more difficult to obtain adequate samples as the GGO components increase, di- 
agnostic accuracy was not statistically different between the two groups. This result might also be related to the use of a cutting needle rather than an aspiration needle. However, nondiagnostic samples were more frequent in lesions with $>90 \%$ GGO components $(n=$ 4) compared with lesions with 50-90\% GGO $(n=1)$. Further study is necessary to evaluate the diagnostic accuracy of core biopsy according to GGO components of the lesions.

Our pneumothorax rate $(18 \%)$ was similar to or lower than the reported rates in the literature $(22-45 \%)$ [30, 33, 41, 42]. Our lower incidence of pneumothorax was likely the result of two main factors. First, we minimized the number of pleural punctures; most procedures were completed with a single puncture. The pneumothorax rate in patients with single punctures is significantly less than in patients with three punctures [33]. Second, there was no intrabiopsy delay for cytopathology evaluation [29]. The prevalence rates of pulmonary hemorrhage and hemoptysis were also in the range of reported complication rates [14-16,
26, 28-32]. These complications did not appear to be clinically significant because none of the patients required treatment.

Currently, no guideline exists for the management of GGO lesions. In our institute, lesions of $<1 \mathrm{~cm}$ in diameter are followed up with thin-section CT 2-3 months later, and those of $\geq 1 \mathrm{~cm}$ in diameter are examined with CT-guided needle biopsy or VATS. Lesions that increase beyond $1 \mathrm{~cm}$ or with a newly developed solid component at follow-up CT also undergo CT-guided needle biopsy or VATS. It is generally acknowledged that pulmonary lesions with a high probability of malignancy that are resectable should proceed directly to surgery because neither a positive nor a negative needle biopsy result is likely to alter management [43]. However, in terms of daily clinical routine, patients may be unwilling to contemplate thoracotomy without preoperative confirmation of malignancy [44-46]. In fact, in our institute many patients with persistent GGO are reluctant to undergo surgical resection without preoperative histologic confir-

TABLE 3: Diagnostic Yield and Accuracy Based on Ground-Glass Opacity

\begin{tabular}{l|c|c|c}
\hline \multirow{2}{*}{\multicolumn{1}{c|}{ Variable }} & \multicolumn{2}{|c|}{ Ground-Glass Opacity } & \multirow{2}{*}{} \\
\cline { 2 - 3 } & $50-90 \%$ & $>90 \%$ & 1.0 \\
\hline Sensitivity (\%) & $91(20 / 22)$ & $93(13 / 14)$ & 1.0 \\
Accuracy (\%) & $92(23 / 25)$ & $91(19 / 21)$ & 0.0072 \\
Lesion diameter (mm) & 22 & 16 & 0.3343 \\
Lesion depth (mm) & 21 & 18 & 0.1628 \\
No. of nondiagnostic & 1 & 4 & \\
samples & & & \\
\hline
\end{tabular}

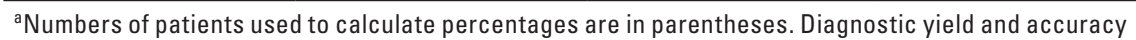
are calculated based on 46 biopsy results excluding five nondiagnostic samples and two samples without confirmative diagnosis due to loss of follow-up.

\section{TABLE 4: Comparison of Histologic Diagnoses from Core Biopsy and Surgery} in Malignant and Premalignant Lesions

\begin{tabular}{l|l|c}
\hline \multicolumn{1}{c|}{ Core Biopsy } & \multicolumn{1}{|c}{ Surgical Biopsy } & No. of Lesions \\
\hline $\begin{array}{l}\text { Concordant } \\
\begin{array}{l}\text { Adenocarcinoma with BAC } \\
\text { feature }\end{array}\end{array}$ & $\begin{array}{l}\text { Adenocarcinoma with BAC } \\
\text { feature } \\
\text { BAC }\end{array}$ & \begin{tabular}{c}
18 \\
\hline BACal
\end{tabular} \\
$\begin{array}{l}\text { Discordant } \\
\text { BAC }\end{array}$ & $\begin{array}{l}\text { Adenocarcinoma with BAC } \\
\text { feature }\end{array}$ & 24 \\
AAH & $\begin{array}{l}\text { BAC } \\
\text { Chronic inflammation }\end{array}$ & 1 \\
AAH & & 9 \\
\hline Total & & 33 \\
\hline Total overall & & 9 \\
\hline
\end{tabular}

Note- $\mathrm{BAC}=$ bronchoalveolar carcinoma, $\mathrm{AAH}=$ atypical adenomatous hyperplasia. mation. Most GGO lesions are detected in an asymptomatic screening population, and they typically show no significant interval change on follow-up imaging. A good chance of a diagnosis of lung cancer with GGO is another possible explanation for this reluctance. Although VATS biopsy can provide larger tissue specimens and improve accuracy, the morbidity and length of stay are greater than those with core biopsy; thus, the decision as to whether to perform core biopsy or surgical resection should be determined on an individual basis, considering not only the clinical and radiologic features but also the views of the patient.

Our study has several limitations. First, the follow-up duration was shorter than that of other studies. Pulmonary nodules are considered benign if they show no growth for 2 years; however, GGO lesions such as atypical adenomatous hyperplasia or BAC may persist without an interval change long after a 2 -year follow-up period $[47,48]$. There is a possibility of a missed lung cancer in patients without confirmative diagnosis; thus, we arranged for these patients to be followed up with serial CT. Second, to interpret the performance of any diagnostic procedure, the pretest probability of malignancy should be known. Our study population included patients with persistent GGO and a high incidence of malignancy; thus, the overall prevalence of malignancy might be higher than that of solitary pulmonary nodules encountered in a clinical setting [49]. Third, in patients who are candidates for surgery, the role of preoperative imaging-guided biopsy is unclear. However, this does not change the focus of this study, which was to determine the diagnostic accuracy of CTguided core biopsy for GGO lesions.

In conclusion, the results of this study suggest that CT-guided core biopsy of pulmonary GGO lesions can yield high diagnostic accuracy and acceptable complication rates approaching those of solid lesions. It can be a safe and accurate diagnostic alternative to surgical biopsy, especially when patients are unwilling to undergo surgical biopsy or take surgical risks.

\footnotetext{
References

1. Kaneko M. Peripheral lung cancer: screening and detection with low-dose spiral CT versus radiography. Radiology 1996; 201:798-802

2. Sone S, Takashima S, Li F, et al. Mass screening for lung cancer with mobile spiral computed tomography scanner. Lancet 1998; 351:1242-1245

3. Henschke CI, McCauley DI, Yankelevitz DF, et al. Early Lung Cancer Action Project: overall design and findings from baseline screening. Lancet 1999 ; 354:99-105
} 
4. Kushihashi T. Bronchioloalveolar adenoma of the lung: CT-pathologic correlation. Radiology 1994; 193:789-793

5. Collins J, Stern EJ. Ground-glass opacity at CT: the ABCs. AJR 1997; 169:355-367

6. Park CM, Goo JM, Lee HJ, et al. CT findings of atypical adenomatous hyperplasia in the lung. $\mathrm{Ko}$ rean J Radiol 2006; 7:80-86

7. Henschke CI, Yankelevitz DF, Mirtcheva R. CT screening for lung cancer: frequency and significance of part-solid and nonsolid nodules. AJR 2002; 178:1053-1057

8. Li F, Sone S, Abe H, MacMahon H, Doi K. Malignant versus benign nodules at $\mathrm{CT}$ screening for lung cancer: comparison of thin-section CT findings. Radiology 2004; 233:793-798

9. Nakata M, Saeki H, Takata I, et al. Focal groundglass opacity detected by low-dose helical CT. Chest 2002; 121:1464-1467

10. vanSonnenberg E, Lin AS, Deutsch AL, Mattrey RF. Percutaneous biopsy of difficult mediastinal, hilar, and pulmonary lesions by computed-tomographic guidance and a modified coaxial technique. Radiology 1983; 148:300-302

11. Khouri NF, Stitik FP, Erozan YS, et al. Transthoracic needle aspiration biopsy of benign and malignant lung lesions. AJR 1985; 144:281-288

12. Stanley JH. Lung lesions: cytologic diagnosis by fine-needle biopsy. Radiology 1987; 162:389-391

13. Westcott JL. Percutaneous transthoracic needle biopsy. Radiology 1988; 169:593-601

14. Yankelevitz DF, Henschke CI, Koizumi JH, Altorki NK, Libby D. CT-guided transthoracic needle biopsy of small solitary pulmonary nodules. Clin Imaging 1997; 21:107-110

15. Larscheid RC. Percutaneous transthoracic needle aspiration biopsy: a comprehensive review of its current role in the diagnosis and treatment of lung tumors. Chest 1998; 114:704-709

16. Tsukada H, Satou T, Iwashima A, Souma T. Diagnostic accuracy of CT-guided automated needle biopsy of lung nodules. AJR 2000; 175:239-243

17. Austin JH, Muller NL, Friedman PJ, et al. Glossary of terms for CT of the lungs: recommendations of the Nomenclature Committee of the Fleischner Society. Radiology 1996; 200:327-331

18. Travis WD, Brambilla E, Muller-Hermelink HK, Harris CC. World Health Organization classification of tumours: pathology and genetics of tumours of the lung, pleura, thymus and heart. Lyon, France: International Agency for Research on Cancer Press, 2004

19. Kaneko M, Kusumoto M, Kobayashi T, et al. Computed tomography screening for lung carcinoma in Japan. Cancer 2000; 89:2485-2488

20. MacMahon H, Austin JHM, Gamsu G, et al. Guidelines for management of small pulmonary nodules detected on CT scans: a statement from the Fleischner Society. Radiology 2005; 237:395-400

21. Travis WD, Garg K, Franklin WA, et al. Evolving concepts in the pathology and computed tomography imaging of lung adenocarcinoma and bronchioloalveolar carcinoma. J Clin Oncol 2005; 23:3279-3287

22. Suzuki K, Yokose T, Yoshida J, et al. Prognostic significance of the size of central fibrosis in peripheral adenocarcinoma of the lung. Ann Thorac Surg 2000; 69:893-897

23. Oh J-Y, Kwon S-Y, Yoon H-I, et al. Clinical significance of a solitary ground-glass opacity (GGO) lesion of the lung detected by chest CT. Lung Cancer 2007; 55:67-73

24. Nakamura H, Saji H, Ogata A, Saijo T, Okada S, Kato H. Lung cancer patients showing pure ground-glass opacity on computed tomography are good candidates for wedge resection. Lung Cancer 2004; 44:61-68

25. Shimizu K, Ikeda N, Tsuboi M, Hirano T, Kato H. Percutaneous CT-guided fine needle aspiration for lung cancer smaller than $2 \mathrm{~cm}$ and revealed by ground-glass opacity at CT. Lung Cancer 2006; 51:173-179

26. Westcott JL, Rao N, Colley DP. Transthoracic needle biopsy of small pulmonary nodules. Radiology 1997; 202:97-103

27. Wang SE, Nieberg RK. Fine needle aspiration cytology of sclerosing hemangioma of the lung, a mimicker of bronchioloalveolar carcinoma. Acta Cytol 1986; 30:51-54

28. Greif J, Marmur S, Schwarz Y, Man A, Staroselsky AN. Percutaneous core cutting needle biopsy compared with fine-needle aspiration in the diagnosis of peripheral lung malignant lesions: results in 156 patients. Cancer 1998; 84:144-147

29. Klein JS, Salomon G, Stewart EA. Transthoracic needle biopsy with a coaxially placed 20-gauge automated cutting needle: results in 122 patients. Radiology 1996; 198:715-720

30. Li H, Boiselle PM, Shepard JO, Trotman-Dickenson B, McLoud TC. Diagnostic accuracy and safety of CT-guided percutaneous needle aspiration biopsy of the lung: comparison of small and large pulmonary nodules. AJR 1996; 167:105-109

31. Laurent F, Latrabe V, Vergier B, Montaudon M, Vernejoux JM, Dubrez J. CT-guided transthoracic needle biopsy of pulmonary nodules smaller than $20 \mathrm{~mm}$ : results with an automated 20-gauge coaxial cutting needle. Clin Radiol 2000; 55:281-287

32. Wallace MJ, Krishnamurthy S, Broemeling LD, et al. CT-guided percutaneous fine-needle aspiration biopsy of small $(<$ or $=1-\mathrm{cm})$ pulmonary lesions. Radiology 2002; 225:823-828

33. Ohno Y, Hatabu H, Takenaka D, et al. CT-guided transthoracic needle aspiration biopsy of small ( $\leq 20 \mathrm{~mm})$ solitary pulmonary nodules. AJR 2003;
180:1665-1669

34. Montaudon M, Latrabe V, Pariente A, Corneloup $\mathrm{O}$, Begueret H, Laurent F. Factors influencing accuracy of CT-guided percutaneous biopsies of pulmonary lesions. Eur Radiol 2004; 14:1234-1240

35. Lucidarme O. Intrapulmonary lesions: percutaneous automated biopsy with a detachable, 18-gauge, coaxial cutting needle. Radiology 1998; 207:759-765

36. Goralnik CH, O’Connell DM, el Yousef SJ, Haaga JR. CT-guided cutting-needle biopsies of selected chest lesions. AJR 1988; 151:903-907

37. Staroselsky AN. Additional information from percutaneous cutting needle biopsy following fineneedle aspiration in the diagnosis of chest lesions. Chest 1998; 113:1522-1525

38. Arakawa H, Nakajima Y, Kurihara Y, Niimi H, Ishikawa T. CT-guided transthoracic needle biopsy: a comparison between automated biopsy gun and fine needle aspiration. Clin Radiol 1996; 51:503-506

39. Pappa VI, Hussain HK, Reznek RH, et al. Role of image-guided core-needle biopsy in the management of patients with lymphoma. J Clin Oncol 1996; 14:2427-2430

40. Travis WD, Garg K, Franklin WA, et al. Bronchioloalveolar carcinoma and lung adenocarcinoma: the clinical importance and research relevance of the 2004 World Health Organization pathologic criteria. J Thorac Oncol 2006; 1:S13-S19

41. vanSonnenberg E, Casola G, Ho M, et al. Difficult thoracic lesions: CT-guided biopsy experience in 150 cases. Radiology 1988; 167:457-461

42. Kazerooni EA, Lim FT, Mikhail A, Martinez FJ. Risk of pneumothorax in CT-guided transthoracic needle aspiration biopsy of the lung. Radiology 1996; 198:371-375

43. Winer-Muram HT. The solitary pulmonary nodule. Radiology 2006; 239:34-49

44. Murphy JM, Gleeson FV, Flower CD. Percutaneous needle biopsy of the lung and its impact on patient management. World J Surg 2001; 25:373-379

45. Cummings SR, Lillington GA, Richard RJ. Managing solitary pulmonary nodules: the choice of strategy is a "close call." Am Rev Respir Dis 1986; 134:453-460

46. Ost D, Fein A. Evaluation and management of the solitary pulmonary nodule. Am J Respir Crit Care Med 2000; 162:782-787

47. Hasegawa M, Sone S, Takashima S, et al. Growth rate of small lung cancers detected on mass CT screening. Br J Radiol 2000; 73:1252-1259

48. Aoki T, Nakata H, Watanabe H, et al. Evolution of peripheral lung adenocarcinomas: CT findings correlated with histology and tumor doubling time. AJR 2000; 174:763-768

49. Cummings SR, Lillington GA, Richard RJ. Estimating the probability of malignancy in solitary pulmonary nodules: a Bayesian approach. Am Rev Respir Dis 1986; 134:449-452 